International Journal of Bifurcation and Chaos, Vol. 8, No. 12 (1998) 2433-2438

(c) World Scientific Publishing Company

\title{
CONTROL OF CHAOS USING SAMPLED-DATA FEEDBACK CONTROL
}

\author{
TAO YANG and LEON O. CHUA \\ Electronics Research Laboratory and \\ Department of Electrical Engineering and Computer Sciences, \\ University of California at Berkeley, \\ Berkeley, CA 94720, USA
}

Received June 14, 1998; Revised August 5, 1998

\begin{abstract}
In this paper we present a theory for control of chaotic systems using sampled data. The output of the chaotic system is sampled at a given sampling rate and the sampled output is used by a feedback subsystem to construct a control signal, which is held constant by a holding subsystem. Hence, during each control iteration, the control input remains unchanged. Theoretical results on the asymptotic stability of the resulting controlled chaotic systems are presented. Numerical experimental results via Chua's circuit are used to verify the theoretical results.
\end{abstract}

\section{Introduction}

The control of chaos by sampled data has been studied and observed in experiments [Yang \& Chua, 1997a, 1997b; Panas et al., 1998; Dedieu \& Ogorzalek, 1994]. Previous results used the sampled data to change the state variables of the chaotic system "impulsively". In this paper, we redesign the controller such that the control signal, which is constructed from the sampling sequence of the output of the chaotic system, is fed into the chaotic system as a control input. In this sampled-data feedback control scheme, the state variables of the chaotic system are subject to continuous changes instead of "impulsive" changes. Unlike most of the previous results where the control input is constructed by the continuous observations of the output of the chaotic system, the controller presented in this paper uses the samples of the output of the chaotic system to construct control signals.

The main motivation for controlling chaos using sampled data is to exploit well-developed digital control techniques. In a digital controller, the output of the chaotic system is sampled and the sampled data is used to construct the appropriate control signals. Assuming that a finite time duration is needed by a digital processor to calculate the control signals, then the sampling frequency is limited by this time duration. On the other hand, a fast sampling device is usually more expensive than a slow one. It is important therefore to develop a theory to predict the performance of the controlled chaotic system with a given sampling rate.

The authors of [Dedieu \& Ogorzalek, 1994] had presented some experimental results for controlling chaotic systems to referenced trajectories by using only sampled values. Although it is widely believed that the control of continuous chaotic systems by using digital controllers is possible, so far, there exists no theoretical results to guarantee the asymptotic stability of such controlled chaotic systems. In this paper, we present theoretical results which guarantee the asymptotic stability of sampled-data feedback control of chaotic systems.

The organization of this paper is as follows. In Sec. 2, the structure and theory of the sampled-data feedback controller are presented. In Sec. 3, the sampled-data feedback control of a typical chaotic 
system (Chua's circuit) is given. In Sec. 4, some concluding remarks are given.

\section{Control of Chaos Using Sampled Data}

In this section we present the structure and the theory of a sampled-data feedback controller.

\subsection{Structure of control system}

The proposed structure of a chaotic control system with sampled data is shown in Fig. 1. The state variables of the chaotic system are observed (measured) by transducers and the result is used to construct the output signal $\mathbf{y}(t)=D \mathbf{x}(t)$ where $D$ is a constant matrix to be defined below. The output $\mathbf{y}(t)$ is then sampled by the sampling block to obtain $\mathbf{y}(k)=D \mathbf{x}(k)$ at discrete moments $k \Delta$, where $k=0,1,2, \ldots$, and $\Delta$ is the sampling duration. Then $D \mathbf{x}(k)$ is used by the controller to calculate the control signal $\mathbf{u}(k)$. During the time interval $[k \Delta,(k+1) \Delta)$, the output of the holding block is $\mathbf{u}(k)$, which is fed back into the chaotic system as a fixed control input during the entire time slot $[k \Delta,(k+1) \Delta)$.

\subsection{Theory of stability}

Consider a chaotic dynamic system modeled by the state equation

$$
\dot{\mathbf{x}}=\mathbf{f}(\mathbf{x})
$$

where $\mathbf{x} \in \Re^{n}$ is the state variable, $\mathbf{f}: \Re^{n} \mapsto \Re^{n}$ is a nonlinear function and $\mathbf{f}(\mathbf{0})=\mathbf{0}$.

The controlled chaotic system is defined by

$$
\left\{\begin{array}{l}
\dot{\mathbf{x}}(t)=\mathbf{f}(\mathbf{x}(t))+B \mathbf{u}(k), t \in[k \Delta,(k+1) \Delta) \\
\mathbf{u}(k+1)=C \mathbf{u}(k)+D \mathbf{x}(k), k=0,1,2, \ldots
\end{array}\right.
$$

where $\mathbf{u} \in \Re^{m}, B \in \Re^{n} \times \Re^{m}, C \in \Re^{m} \times \Re^{m}$, $D \in \Re^{m} \times \Re^{n}$, and $t \in \Re_{+} ; \mathbf{x}(k)$ is the sampled value of $\mathbf{x}(t)$ at $t=k \Delta ; \Delta$ is the sampling duration. Observe that since $\mathbf{f}(\mathbf{0})=\mathbf{0},\left(\begin{array}{l}\mathbf{x} \\ \mathbf{u}\end{array}\right)=\left(\begin{array}{l}\mathbf{0} \\ \mathbf{0}\end{array}\right)$ is an equilibrium point of the system in Eq. (2). The asymptotic stability of this equilibrium point can be determined from that of the trivial solution of the associated linearized system:

$$
\left\{\begin{array}{l}
\dot{\mathbf{x}}=A \mathbf{x}+B \mathbf{u}(k), t \in[k \Delta,(k+1) \Delta) \\
\mathbf{u}(k+1)=C \mathbf{u}(k)+D \mathbf{x}(k), k=0,1,2, \ldots
\end{array}\right.
$$

where $A \in \Re^{n} \times \Re^{n}$ is given by

$$
A \triangleq \frac{\partial \mathbf{f}(0)}{\partial \mathbf{x}}
$$

Definition 1. An $n \times n$ matrix $\Gamma$ is said Schur stable if, and only if, all eigenvalues of $\Gamma$ lie in the unit disc centered at the origin.

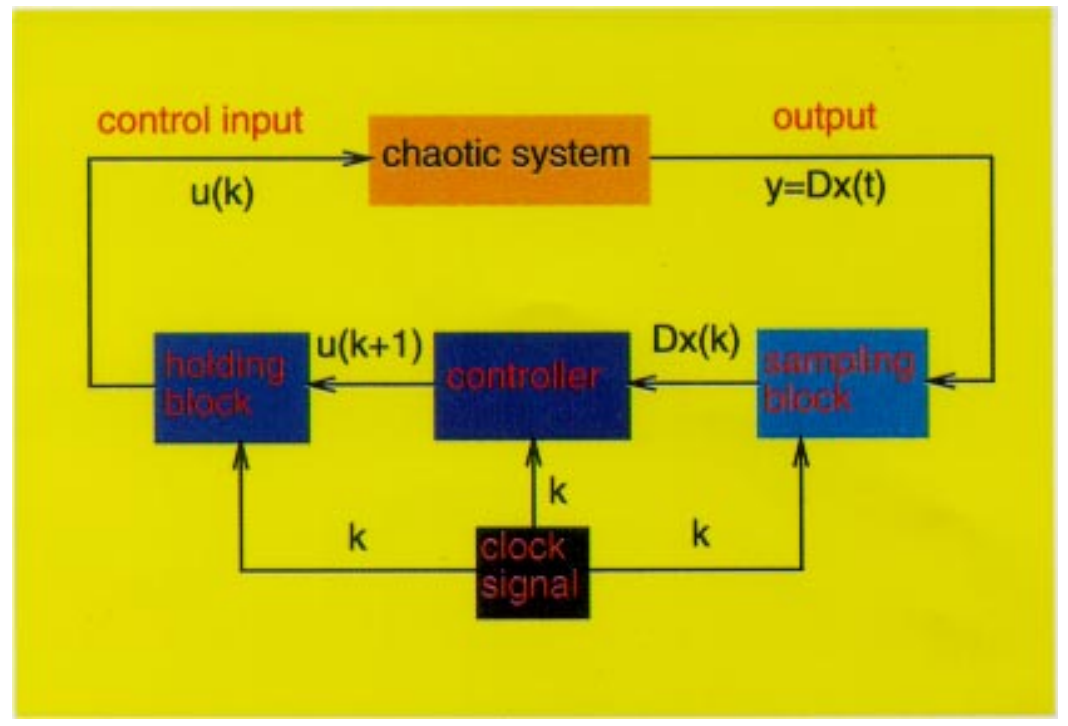

Fig. 1. Structure of a proposed sampled-data chaotic control system. 
Lemma 1. Suppose $\mathbf{f} \in C^{1}\left[\Re^{n}, \Re^{n}\right]$, then the equilibrium point $(\mathbf{x}, \mathbf{u})=(\mathbf{0}, \mathbf{0})$ of the controlled chaotic system (2) is uniformly asymptotically stable if the associated matrix

$$
H \triangleq\left(\begin{array}{cc}
e^{A \Delta} & \left(\int_{0}^{\Delta} A^{\Delta-\tau} d \tau\right) B \\
D & C
\end{array}\right)
$$

is Schur stable.

Proof. The proof follows directly from Theorem 5.1 of [Ye et al., 1998], which assumes that $\Delta=1$. The generalization for arbitrary $\Delta$, hence, follows easily.

Lemma 1 is not practical because it involves integration of matrices. The following theorem gives a more explicit criterion for the stability of the controlled chaotic system.

Theorem 1. Suppose $\mathbf{f} \in C^{1}\left[\Re^{n}, \Re^{n}\right]$ and $A$ is nonsingular, then the equilibrium point $(\mathbf{x}, \mathbf{u})=$ $(\mathbf{0}, \mathbf{0})$ of the controlled chaotic system (2) is uniformly asymptotically stable if the spectral radius $\rho(T)$ of the matrix

$$
T \triangleq\left(\begin{array}{cc}
e^{A \Delta} & A^{-1} e^{A \Delta} B-A^{-1} B \\
D & C
\end{array}\right)
$$

is less than unity; i.e. $\rho(T)<1$.

Proof. Observe that

$$
\begin{aligned}
\left(\int_{0}^{\Delta} A^{\Delta-\tau} d \tau\right) B & =\left(-\int_{0}^{\Delta} A^{\Delta-\tau} d(\Delta-\tau)\right) B \\
& =\left(\int_{0}^{\Delta} A^{t} d t\right) B \\
& =\left(\left.A^{-1} e^{A t}\right|_{0} ^{\Delta}\right) B \\
& =A^{-1}\left(e^{A \Delta}-I\right) B
\end{aligned}
$$

where $t=\Delta-\tau$ and $I \in \Re^{n} \times \Re^{n}$ is the identity matrix. Substituting (7) into (5), obtain $T=H$. Since the spectral radius $\rho(T)<1$, we know that $T$ is Schur stable, and the theorem therefore follows from Lemma 1.

\section{Control of Chua's Circuit with Sampled Data}

In this example, we use Chua's oscillator [Madan,
1993], which is shown in Fig. 2(a), as the chaotic system. The state equation of the Chua's oscillator is given by

$$
\left\{\begin{array}{l}
\frac{d v_{1}}{d t}=\frac{1}{C_{1}}\left[G\left(v_{2}-v_{1}\right)-f\left(v_{1}\right)\right] \\
\frac{d v_{2}}{d t}=\frac{1}{C_{2}}\left[G\left(v_{1}-v_{2}\right)+i_{3}\right] \\
\frac{d i_{3}}{d t}=\frac{1}{L}\left[-v_{2}-R_{0} i_{3}\right]
\end{array}\right.
$$

where $G=1 / R$ and $f\left(v_{1}\right)$ is the piecewise linear $v-i$ characteristic of Chua's diode, as shown in Fig. 2(b) and can be expressed explicitly by

$$
f\left(v_{1}\right)=G_{b} v_{1}+\frac{1}{2}\left(G_{a}-G_{b}\right)\left(\left|v_{1}+E\right|-\left|v_{1}-E\right|\right)
$$

where $E$ is the breakpoint voltage of Chua's diode.

In this example, let us assume $R_{0}=0$ in (8). The dimensionless version of the resulting Chua's circuit is given by

$$
\left\{\begin{array}{l}
\dot{x}=\alpha(y-x-f(x)) \\
\dot{y}=x-y+z \\
\dot{z}=-\beta y
\end{array}\right.
$$

where $x=v_{1} / E, y=v_{2} / E, z=i_{3} /(E G), \alpha=$ $C_{2} / C_{1}, \beta=C_{2} R^{2} / L$, and

$$
f(x)=b x+\frac{1}{2}(a-b)(|x+1|-|x-1|)
$$

where $a=R G_{a}$ and $b=R G_{b}$. To control Chua's circuit to the origin, let us calculate

$$
A=\left.\frac{\partial \mathbf{f}}{\partial \mathbf{x}}\right|_{\mathbf{x}=\mathbf{0}}=\left(\begin{array}{ccc}
-\alpha(1+a) & \alpha & 0 \\
1 & -1 & 1 \\
0 & -\beta & 0
\end{array}\right) \text {. }
$$

Let us choose the parameters as follows: $\alpha=$ $10, \beta=14.87, a=-1.27$ and $b=-0.68$, and

$$
\begin{aligned}
& B=\left(\begin{array}{lll}
1 & 0 & 0 \\
0 & 0 & 0 \\
0 & 0 & 0
\end{array}\right), \quad C=\left(\begin{array}{ccc}
0.8 & 0 & 0 \\
0 & 0 & 0 \\
0 & 0 & 0
\end{array}\right), \\
& D=\left(\begin{array}{ccc}
-0.6 & 0 & 0 \\
0 & 0 & 0 \\
0 & 0 & 0
\end{array}\right), \Delta=5 \times 10^{-3} .
\end{aligned}
$$




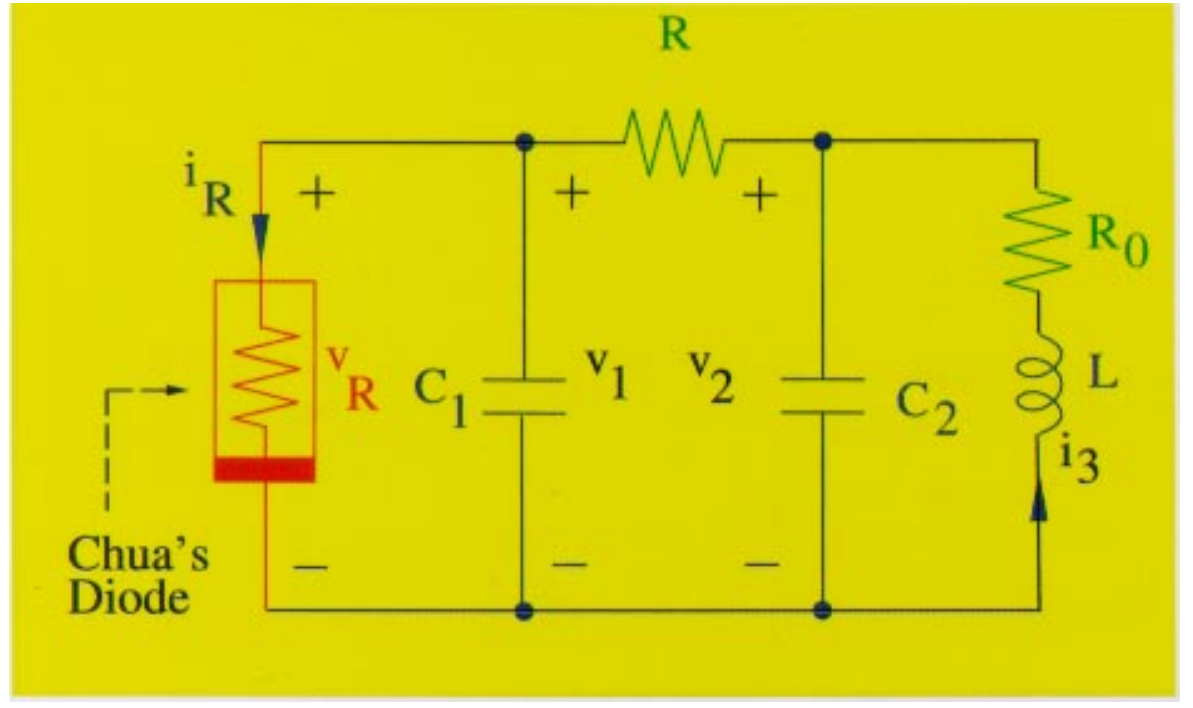

(a)

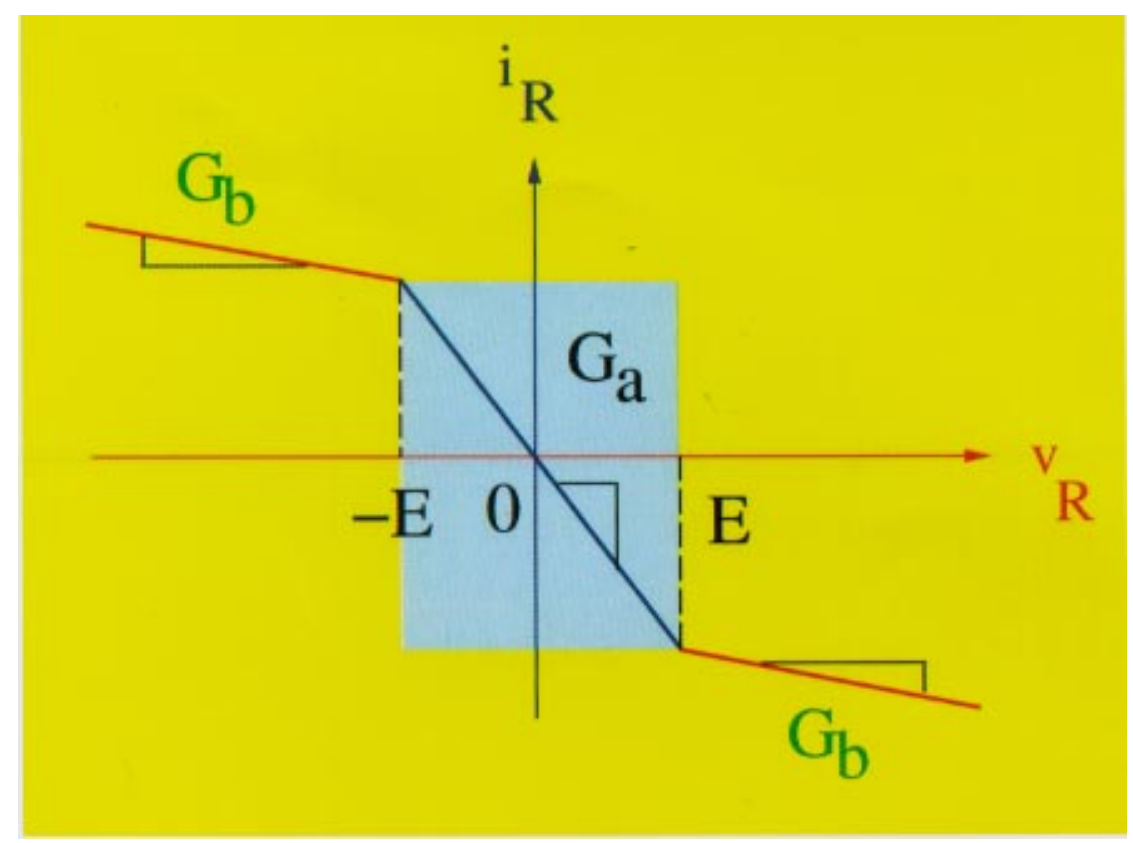

(b)

Fig. 2. (a) Chua's circuit. (b) Nonlinear $v-i$ characteristic of Chua's diode.

Using the above parameters, the controlled chaotic system is given by

$$
\begin{gathered}
\left\{\begin{array}{l}
\dot{x}=\alpha(y-x-f(x))+u_{1}(k) \\
\dot{y}=x-y+z \\
\dot{z}=-\beta y
\end{array}\right. \\
u_{1}(k+1)=0.8 u_{1}(k)-0.6 x(k), k=0,1,2, \ldots
\end{gathered}
$$

The corresponding matrix $T$ in (6) is given by

$$
T=\left(\begin{array}{cccccc}
1.0137 & -0.0502 & 0.0001 & 0.0050 & 0 & 0 \\
0.0050 & -0.9950 & 0.0050 & 0.0000 & 0 & 0 \\
-0.0002 & -0.0742 & 0.9998 & 0.0000 & 0 & 0 \\
-0.6000 & 0 & 0 & 0.8000 & 0 & 0 \\
0 & 0 & 0 & 0 & 0 & 0 \\
0 & 0 & 0 & 0 & 0 & 0
\end{array}\right) .
$$

The eigenvalues and their norms of the above matrix $T$ are listed in Table 1. 


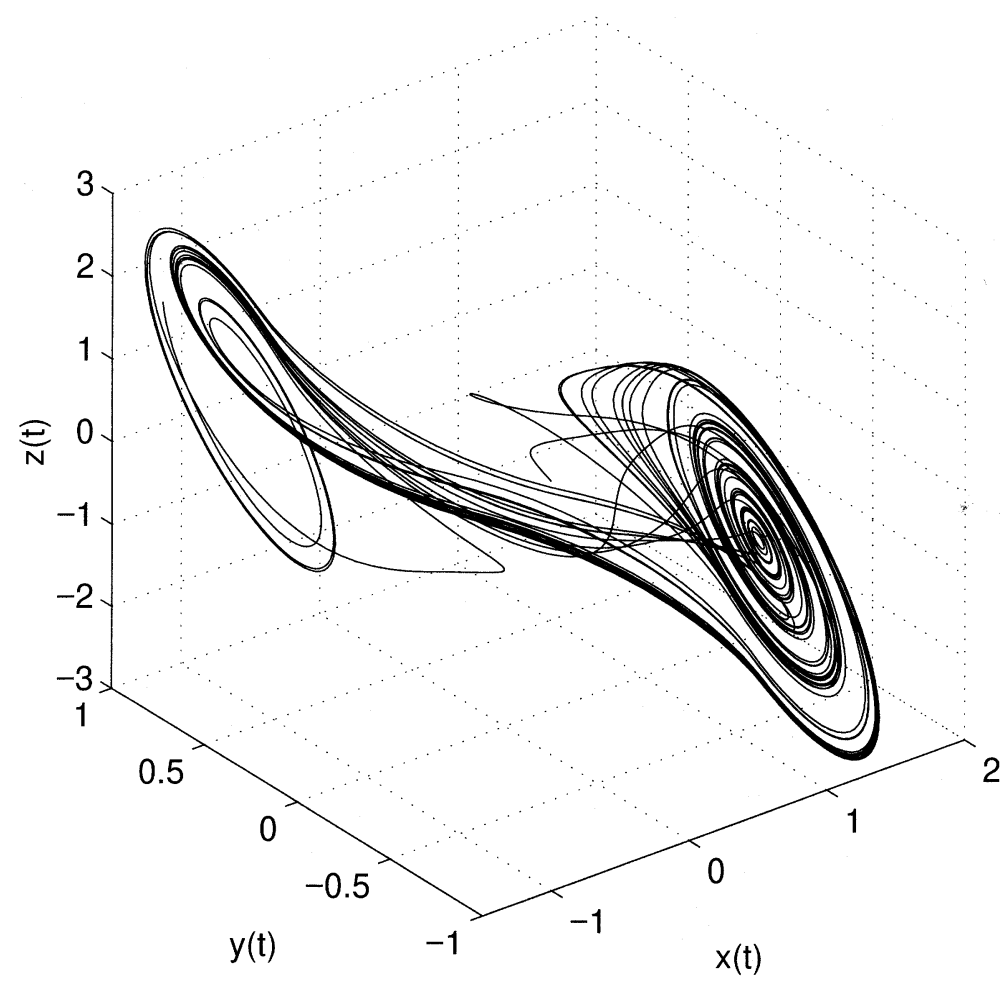

(a)

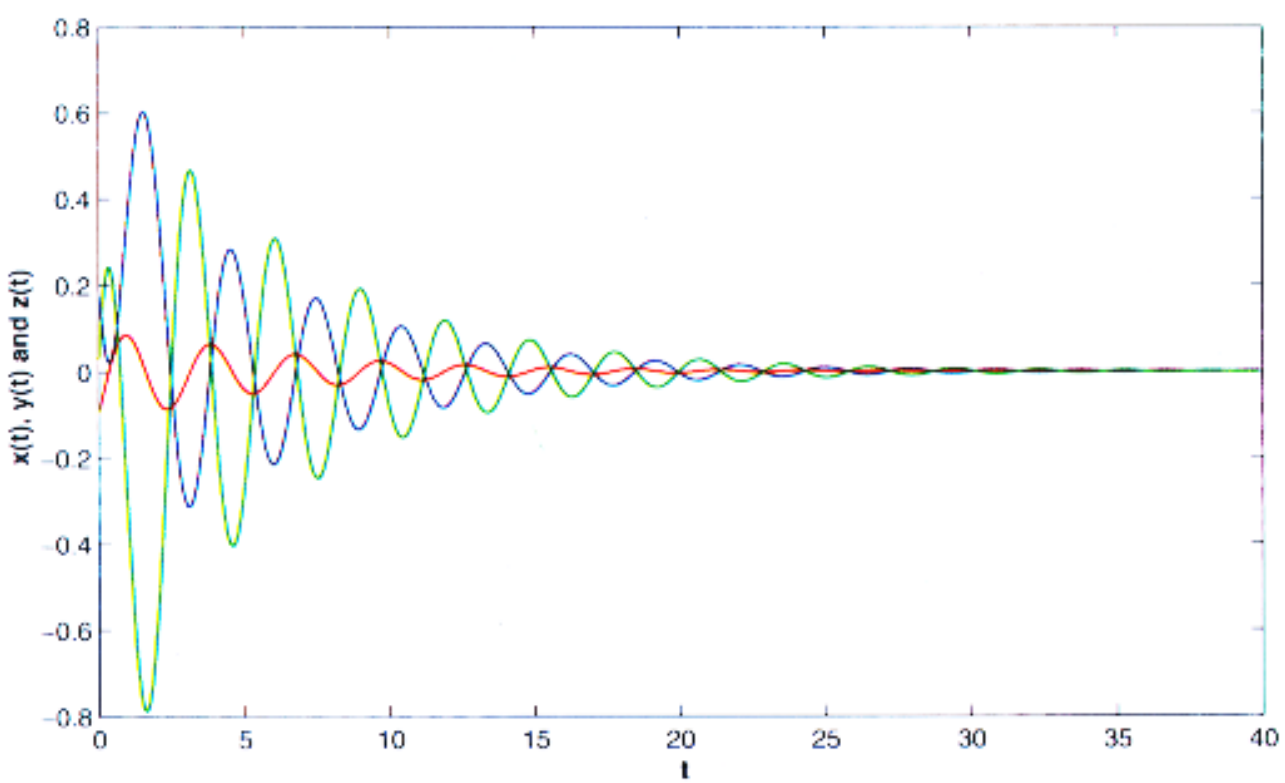

(b)

Fig. 3. Controlling Chua's circuit to the origin using sampled control signal. (a) The trajectory of Chua's circuit without control. (b) The controlled state variables of Chua's circuit: $x(t)$ (blue), $y(t)$ (red) and $z(t)$ (green). (c) Control signal $u_{1}(k)$. 


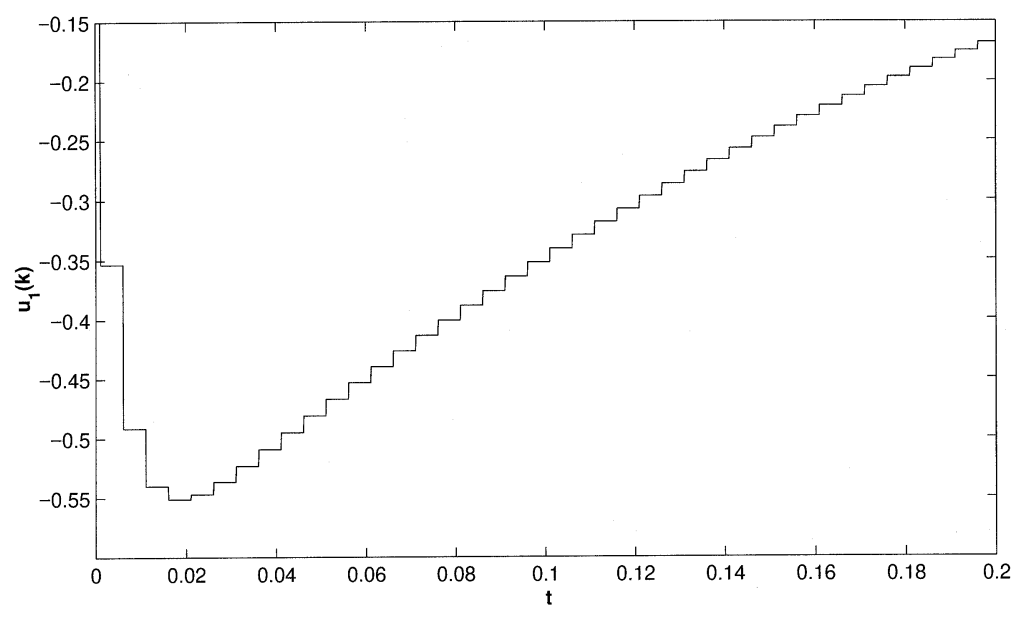

(c)

Fig. 3. (Continued)

Table 1. The eigenvalues and their norms of matrix $T$.

\begin{tabular}{ccc}
\hline Real Part & Imaginary Part & Norm \\
\hline-0.994689 & 0.000000 & 0.994689 \\
0.815117 & 0.000000 & 0.815117 \\
0.998448 & 0.000000 & 0.998448 \\
0.999623 & 0.000000 & 0.999623 \\
0.000000 & 0.000000 & 0.000000 \\
0.000000 & 0.000000 & 0.000000 \\
\hline
\end{tabular}

Since $\rho(T)=0.999623<1$, the controlled system is uniformly asymptotically stable at the origin. The simulation results are shown in Fig. 3. The initial condition for Chua's circuit is $(x(0), y(0), z(0))=$ $(0.2,-0.1,-0.01)$. The initial condition for the controller is $\left(u_{1}(0), u_{2}(0), u_{3}(0)\right)=(0,0,0)$. The sampling rate is $200 \mathrm{~Hz}$. Figure 3(a) shows the trajectory of Chua's circuit without control. Figure 3(b) shows the controlled trajectory, which tends to the origin asymptotically. Figure 3(c) shows the details of the control signal $u_{1}(k)$.

\section{Concluding Remarks}

In the chaotic control community, it has been known for a long time that to control a chaotic system, the sampled data of the output is enough for constructing the control signals. This paper provides the theory of the stability of this method and also provides some constructive guidelines on the design of the controller. Since digital controllers are much more flexible than analog controllers, digital controllers had been widely used in industry. The results presented in this paper give a theoretical basis for designing digital controllers for controlling chaotic systems.

\section{Acknowledgment}

This work is supported in part by the Office of Naval Research under grant numbers N00014-97-10463 and N00014-96-1-0753.

\section{References}

Dedieu, H. \& Ogorzalek, M. [1994] "Controlling chaos in Chua's circuit via sampled inputs," Int. J. Bifurcation and Chaos 4(2), 447-456.

Madan, R. N. (ed.) [1993] Chua's Circuit: A Paradigm for Chaos, World Scientific Series on Nonlinear Science. Series B, Special Theme Issues and Proceedings Vol. 1 (World Scientific, Singapore).

Panas, A. I., Yang, T. \& Chua, L. O. [1998] "Experimental results of impulsive synchronization between two Chua's oscillators," Int. J. Bifurcation and Chaos 8(3), 639-644.

Yang, T. \& Chua, L. O. [1997] "Impulsive control and synchronization of nonlinear dynamical systems and application to secure communication," Int. J. Bifurcation and Chaos 7(3), 645-664.

Yang, T. \& Chua, L. O. [1997] "Impulsive stabilization for control and synchronization of chaotic systems: Theory and application to secure communication," IEEE Trans. Circuits Syst. I: Fundamental Theor. Appl. 44(10), 976-988.

Ye, H., Michel, A. N. \& Hou, L. [1998] "Stability theory for hybrid dynamical systems," IEEE Trans. Automatic Control 43(4), 461-474. 